\title{
Understanding PCle performance for end host networking
}

\author{
Rolf Neugebauer \\ Independent Researcher \\ Yury Audzevich \\ University of Cambridge
}

\author{
Gianni Antichi \\ Queen Mary, University of London \\ Sergio López-Buedo \\ Universidad Autónoma de Madrid
}

\author{
José Fernando Zazo \\ Naudit HPCN
}

Andrew W. Moore

University of Cambridge

\begin{abstract}
In recent years, spurred on by the development and availability of programmable NICs, end hosts have increasingly become the enforcement point for core network functions such as load balancing, congestion control, and application specific network offloads. However, implementing custom designs on programmable NICs is not easy: many potential bottlenecks can impact performance.

This paper focuses on the performance implication of PCIe, the de-facto I/O interconnect in contemporary servers, when interacting with the host architecture and device drivers. We present a theoretical model for PCIe and pcie-bench, an open-source suite, that allows developers to gain an accurate and deep understanding of the PCIe substrate. Using pcie-bench, we characterize the PCIe subsystem in modern servers. We highlight surprising differences in PCIe implementations, evaluate the undesirable impact of PCIe features such as IOMMUs, and show the practical limits for common network cards operating at $40 \mathrm{~Gb} / \mathrm{s}$ and beyond. Furthermore, through pcie-bench we gained insights which guided software and future hardware architectures for both commercial and research oriented network cards and DMA engines.
\end{abstract}

\section{CCS CONCEPTS}

- Networks $\rightarrow$ Network adapters; Network servers; • Hardware $\rightarrow$ Networking hardware; Buses and high-speed links;

Permission to make digital or hard copies of all or part of this work for personal or classroom use is granted without fee provided that copies are not made or distributed for profit or commercial advantage and that copies bear this notice and the full citation on the first page. Copyrights for components of this work owned by others than the author(s) must be honored. Abstracting with credit is permitted. To copy otherwise, or republish, to post on servers or to redistribute to lists, requires prior specific permission and/or a fee. Request permissions from permissions@acm.org. SIGCOMM '18, August 20-25, 2018, Budapest, Hungary

(c) 2018 Copyright held by the owner/author(s). Publication rights licensed to ACM.

ACM ISBN 978-1-4503-5567-4/18/08 ..\$15.00

https://doi.org/10.1145/3230543.3230560

\section{KEYWORDS}

PCIe, reconfigurable hardware, Operating System

ACM Reference Format:

Rolf Neugebauer, Gianni Antichi, José Fernando Zazo, Yury Audzevich, Sergio López-Buedo, and Andrew W. Moore. 2018. Understanding PCIe performance for end host networking. In SIGCOMM '18: SIGCOMM 2018, August 20-25, 2018, Budapest, Hungary. ACM, New York, NY, USA, 15 pages. https://doi.org/10.1145/3230543.3230560

\section{INTRODUCTION}

The idea of end hosts participating in the implementation of network functionality has been extensively explored in enterprise and datacenter networks [6, 7, 25, 49, 56, 58]. Moreover, the disruptive introduction into the market of programmable NICs, alongside the deployment in datacenters of hybrid Xeon and FPGA platforms [15], has boosted the demand for new refined solutions which combine software functions and hardware NIC acceleration to improve end host networking performance [53], flexibility [16], or a combination of both [29]. Several efforts try to leverage end host hardware programmability to improve datacenter scalability $[12,13]$ or specific network functions such as load balancing, application level quality of service and congestion control [1].

In this paper, we show that PCIe, alongside its interaction with the host architecture and device drivers, can significantly impact the performance of network applications. Past research has mostly considered this impact in the context of specific applications such as Remote DMA (RDMA) [24], GPU-accelerated packet processing [17], and optimized KeyValue-Store (KVS) applications [31, 32, 34]. In contrast, we argue that a more generic approach to studying and characterizing PCIe is needed as it has become essential to implement not only specialized, high-performance network functions, but also storage adaptors and custom accelerator cards, such as for machine learning [23]. It is in this context that we introduce a theoretical model for PCIe (§3), design a methodology to characterize PCIe in real systems (§4), describe its implementation (§5), and present the results derived using our approach $(\S 6)$. This permits us to draw several specific conclusions about the way PCIe currently 
operates and the implications this has for operating system and application design (§7). For example, we demonstrate how both NUMA for PCIe devices and IOMMUs can have a negative performance impact on application performance.

The contributions of this paper are:

- We introduce a model of PCIe. The model provides a baseline for expected PCIe throughput for a wide range of PCIe configurations. It also allows to quickly assess the performance impact when iterating through design alternatives for device and device driver interactions, e.g., when implementing custom NIC functionality.

- We outline a methodology, pcie-bench: a combination of micro-benchmarks that systematically measures aspects of performance for both a device's DMA engines and, more crucially, the PCIe root complex ${ }^{1}$.

- We describe two implementations of pcie-bench based on commodity PCIe peripherals: commercially available programmable NICs from Netronome and the latest NetFPGA boards. The implementations are available as open source $^{2}$ to allow for the reproducibility of our results and to enable other practitioners to apply to other systems and architectures.

- We present the first, publicly-disclosed, detailed measurements of PCIe performance across several generations of conventional host-systems; this permits us to trace the evolution of PCIe root complex implementations and highlight some surprising differences between implementations even from the same vendor.

- We discuss the lessons we learned alongside a number of practical use-cases for pcie-bench. From the original need to characterize, debug, and improve implementations, to aid and evaluate the growing area of research of customized solutions enabled by programmable NICs. Furthermore, the results are also directly applicable to the growing number of other high-performance such as specialized accelerator cards.

\section{MOTIVATION}

Traditionally, I/O interconnects in commodity systems, such as PCIe, are not widely studied, in part, because they have worked effectively for many years. It is only as we have I/O devices that approach (and exceed) the capabilities offered by PCIe, e.g., dual-port 40Gb/s network adapters, that we have seen a significant number of hardware-software co-designs ${ }^{3}$

\footnotetext{
${ }^{1}$ The PCIe root complex connects the processors and memory subsystem of a host system with the PCIe switch fabric to individual PCIe devices. Its functionality is similar to the PCI host bridge in older systems.

${ }^{2}$ https://www.pcie-bench.org

${ }^{3}$ Such I/O-aware software design is hardly new. In the past, when faster ATM and $1 \mathrm{~Gb} / \mathrm{s}$ PCI bus adapters were introduced, many driver and kernel redesigns have been proposed $[9,50,60]$.
}

with the constraints of PCIe in mind [16, 29, 30]. Alongside them, many novel software frameworks have also been proposed to avoid the overhead of conventional network stacks $[3,8,46,54]$.

Unfortunately, with 40 and $100 \mathrm{~Gb} / \mathrm{s}$ NICs, PCIe, even in combination with optimized software stacks, is becoming the bottleneck. Moreover, modern network applications with tight latency requirements can be affected by the delays introduced by both the DMA engines in PCIe devices and the PCIe end-host implementation. Finally, the host-side implementation of PCIe in modern x 86 based servers has been changing dramatically, and alternative server architectures are also emerging. We now look at these aspects in more detail.

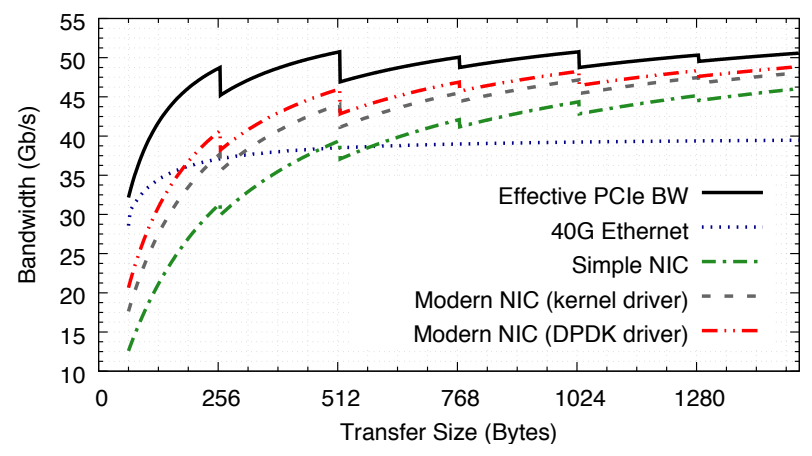

Figure 1: Modeled bidirectional bandwidth of a PCIe Gen $3 \times 8$ link, the achievable throughout of a simplistic NIC and a modern NIC with a typical kernel driver and a DPDK driver.

PCIe impact on network application throughput. A PCIe Gen 3 x8 interface, typically used by $40 \mathrm{~Gb} / \mathrm{s}$ NICs, has a throughput of $62.96 \mathrm{~Gb} / \mathrm{s}$ at the physical layer. However, PCIe protocol overheads reduce the usable bandwidth to around $50 \mathrm{~Gb} / \mathrm{s}$, or significantly less, depending on the PCIe access patterns. Figure 1 shows the effective bi-directional bandwidth achievable for such a device (Effective PCIe $\mathrm{BW})$. The saw-tooth pattern is caused by the packetized structure of the PCIe protocol where the data to be transferred, e.g., a network packet, is broken up into smaller PCIe packets with their own PCIe level headers.

Apart from transferring the packet data itself, a NIC also has to read TX and freelist descriptors, write back RX (and sometimes TX) descriptors and generate interrupts. Device drivers also have to read and update queue pointers on the device. All these interactions are PCIe transactions consuming additional PCIe bandwidth. If we model a very simple NIC, which DMAs individual descriptors for every packet and where the driver reads/writes the queue pointers for 
every packet, we can see a dramatic decrease in achievable bi-directional bandwidth (Simple NIC). Such a device would only achieve $40 \mathrm{~Gb} / \mathrm{s}$ line rate throughput for Ethernet frames larger than 512B. Most modern NICs (and drivers) therefore implement a number of optimizations, such as en-queuing descriptors in batches, pre-fetching descriptors, interrupt moderation, etc. These optimizations increase the complexity of the NIC but are required to achieve acceptable throughput. Modern NIC (kernel driver) in the figure shows the throughput of such a moderately optimized NIC/driver combination with a typical Linux kernel driver. Modern software frameworks, such as DPDK [8], enable further optimizations at the driver level (no NIC hardware changes). By disabling interrupts and polling write-back descriptors in host memory instead of device registers, the number of PCIe transactions is further reduced and the achievable bandwidth is increased (Modern NIC (DPDK driver)). We detail how these models are derived in Section 3. However, Figure 1 shows that when designing custom offloads to programmable NICs, developers and researchers have to be acutely aware of the overheads imposed by PCIe transactions, caused both by the device and the device driver.

PCIe impact on network application latency. We used an ExaNIC [11] to estimate the contribution of PCIe to the overall end-host latency experienced by a network application. We executed a loopback test to measure the total NIC latency (from application to the wire and back) with various sized packets. The test writes a packet to the driver's buffer and measures the latency between when the packet starts to be written to PCIe and when the packet returns. The test utilizes kernel bypass mode, so does not include any kernel overheads. We also modified the ExaNIC firmware, using Exablaze firmware development kit ${ }^{4}$, to measure the PCIe latency contribution.

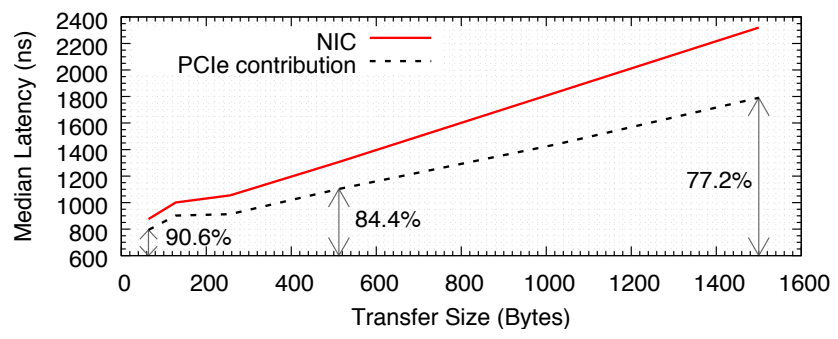

Figure 2: Measurement of NIC PCIe latency.

Figure 2 shows the results from the test. On the ExaNIC, the PCIe subsystem contributes between $77 \%$ of the latency for $1500 B$ packets, increasing to more than $90 \%$ for small

\footnotetext{
${ }^{4} \mathrm{http} / / / \mathrm{www} . e x a b l a z e . c o m / d o c s /$ exanic/user-guide/fdk/fdk/
}

packet sizes. In particular, the overall latency is well within the range which has been shown to have negative impact on performance for several common datacenter applications [63].

Measurement of NIC PCIe latency Figure 2 illustrates that the round trip latency for a $128 \mathrm{~B}$ payload is around $1000 n s$ with PCIe contributing around 900 ns. With $40 \mathrm{~Gb} / \mathrm{s}$ Ethernet at line rate for $128 \mathrm{~B}$ packets, a new packet needs to be received and sent around every 30ns. Assuming that the measured PCIe latency is symmetric, this implies that the NIC has to handle at least 30 concurrent DMAs in each direction to accommodate this latency in order to achieve line rate for $128 \mathrm{~B}$ packets. Given that a NIC also has to issue DMAs for descriptors, the number of in-flight DMAs a NIC has to handle is even higher. Furthermore, as we show in $\S 6.2$, some systems introduce significant variance in latencies, which the NIC's DMA engines also have to accommodate. This not only introduces significant complexity when writing optimized software for programmable NICs but also imposes constraints on the device driver having to supply host buffers in large enough batches for the NIC to consume.

PCIe root complex is evolving. In modern, x86 based servers, the PCIe root complex has seen a rapid evolution in recent years. Specifically, together with the memory controller(s), it has been closely integrated with the CPU cores, enabling tighter integration of PCIe devices with a CPU's caches, e.g., Intel's Data Direct I/O (DDIO) [20]. For multisocket systems, this integration also results in non-uniform memory access (NUMA [28]) for PCIe devices: Some DMA requests may target memory local to the socket the PCIe device is attached to while others need to traverse the CPU interconnect. Finally, most modern systems have an IOMMU [22] interposed in the data path between a PCIe device and the host. The IOMMU performs address-translation for addresses present in PCIe transactions and utilizes an internal Transaction Lookaside Buffer (TLB) as a cache for translated addresses. On a TLB miss, the IOMMU must perform a full page table walk, which may delay the transaction and thus may increase latency and impact throughput. These recent technologies not only impact the overall latency (and bandwidth) of PCIe transactions, but they also introduce variance as transactions are now depending on the temporal state of caches, IOMMU TLB and the characteristics of the CPU interconnects. Furthermore, after years of a relative x86 monoculture in the server market, designs around ARM64 and Power processors have all received mainstream deployment. Each of these architectures have vastly different implementations of the I/O subsystem and most of the associated details are not publicly available, making it hard for researchers to characterize and understand the differences among these server architectures. 
The impact of PCIe on the throughput experienced when data needs to be transferred from hardware to software and vice versa, the predominant contribution of PCIe to the overall NIC latency, the introduction of new technologies in the PCIe root complex, and the mainstream deployment of new server architectures have motivated us to build pcie-bench: to get a better, more detailed and systematic understanding of the PCIe implementation in commodity systems and to help developers to fine tune applications to make more efficient use of the underlying system.

\section{MODELLING PCIE}

As part of pcie-bench we have developed a model of the PCIe specification $[47,57]$. The model allows us to: (1) validate bandwidth measurements from micro-benchmarks against expected bandwidth based on the specification, and (2) model the impact of more complex device/host interactions, such as shown in Figure 1 with the NIC models.

PCIe uses a high-speed serial interconnect based on a point-to-point topology consisting of several serial links (or lanes) between endpoints. It is a protocol with three layers: physical, data link and transaction. While the data link layer (DLL) implements error correction, flow control and acknowledgments, the transaction layer turns user application data, or completion data, into PCIe transactions using Transaction Layer Packets (TLPs).

Most available $40 \mathrm{~Gb} / \mathrm{s}$ NICs today have a PCIe Gen 3 interface with 8 lanes. Each lane offers 8 GT/s (Giga Transactions per second) using a $128 \mathrm{~b} / 130 \mathrm{~b}$ encoding, resulting in $8 \times 7.87 \mathrm{~Gb} / \mathrm{s}=62.96 \mathrm{~Gb} / \mathrm{s}$ at the physical layer. The DLL adds around $8-10 \%$ of overheads due to flow control and acknowledgment messages, leaving around $57.88 \mathrm{~Gb} / \mathrm{s}$ available at the TLP layer ${ }^{5}$. For each transaction, the physical layer adds $2 B$ of framing and the DLL adds a $6 B$ header.

At the transport layer, the standard defines a number of different TLP types. For the purpose of this paper, the relevant packet types are: Memory Read (MRd), Memory Write (MWr), and Completion with Data (CplD). TLPs have a common header, a type specific header, and an optional trailing digest. The common header contains information, such as the type of TLP and the TLP length, and is $4 B$ in size. The header of MWr and MRd TLPs is $12 B$ long (assuming 64bit addressing) while for CplD TLPs it is $8 B$. The maximum amount of data carried in a single TLP (Maximum Payload Size, or MPS) is negotiated between the peers and the optional $4 B$ digest contains an end-to-end CRC (ECRC). Typical values for the MPS are $256 B$ or $512 B$.

\footnotetext{
${ }^{5}$ The DLL overhead depends on the transaction rate and implementation details. The value of $57.88 \mathrm{~Gb} / \mathrm{s}$ was derived using recommended values from the PCIe specification [47].
}

PCIe MWr transactions are simple posted transactions and the number of bytes transferred by a DMA write of size $s z$ can be calculated with:

$$
B_{t x}=\lceil s z / M P S\rceil \times M W r_{-} H d r+s z
$$

where $M W r \_H d r$ is $24 B$ ( $2 B$ framing, $6 B$ DLL header, $4 B$ TLP header, and $12 B$ MWr header). In contrast, PCIe memory reads (MRd), e.g., a device reading data from the host, require two TLP types: MRd TLPs and CplD TLPs. A MRd TLP is sent to request data from the peer, then the data is returned via one or more CplD TLPs. Thus, PCIe memory reads consume bandwidth in both directions. A MRd request may only request data up a certain amount (Maximum Read Request Size, or MRRS), negotiated between the peers. A typical value for the MRRS is $512 B$. The data returned via CplD TLPs is bounded by the MPS. The number of bytes consumed for a DMA read of size $s z$ is then:

$$
\begin{aligned}
& B_{t x}=\lceil s z / M R R S\rceil \times M R d \_H d r+s z \\
& B_{r x}=\lceil s z / M P S\rceil \times C p l D \_H d r+s z
\end{aligned}
$$

with MRd_Hdr being 24B and CPL_Hdr 20B.

The PCIe configuration of a device, e.g., PCIe Gen 3 device with 8 lanes (x8), provides us with the available bandwidth and the values for MPS and MRRS. With the formula above, we can then calculate the effective bandwidth for different transfer sizes $s z$. The graph Effective PCIe BW in Figure 1 was calculated using this model assuming a PCIe Gen 3 x8 device, $M P S=256 B$, and $M R R S=512 B$ using 64bit addressing. The saw-tooth pattern shows the overhead of the additional DLL/TLP headers for every MPS bytes of data, with the overhead being higher for smaller transfer sizes. The graph also shows the impact of MRRS as the additional MRd TLPs consume bandwidth which otherwise would be usable by MWr transactions.

The model also allows us to calculate the overhead for more complex device/host interactions. For example, for the graph of the Simple NIC in Figure 1 we calculate the PCIe bandwidth used both for packet send (TX) and receive (RX) of a simple NIC. For TX, the driver writes updates the TX queue tail pointer on the device ( $4 B$ PCIe write). The device then DMAs the descriptor (16B PCIe read) and, subsequently, the packet buffer. After transmission, the device generates an interrupt ( $4 B$ PCIe write) and the driver reads the TX queue head pointer ( $4 B$ PCIe read). For RX, the driver updates the $\mathrm{RX}$ queue tail pointer to enqueue a buffer on the freelist $(4 B$ PCIe write). The device then DMAs the freelist descriptor (16B PCIe read), DMAs the packet (PCIe write) and the RX descriptor (16B PCIe write) to the host and generates an interrupt ( $4 B$ PCIe write). Finally, the driver reads the RX queue head pointer ( $4 B$ PCIe read). The model then calculates the overhead for each of these transactions and computes the achievable bandwidth. 
The NIC model described above is very simplistic and all modern NICs deploy a variety of optimizations. For example, the Intel Niantic NIC [19] DMAs batches of up to 40 TX descriptors from the host and may write batches up to 8 TX descriptors back to the host. Optimized NIC (kernel driver) in Figure 1 shows the performance impact of these optimizations over the simple NIC model. Throughput can be further improved by driver changes, as shown by Optimized NIC (DPDK driver) in the same figure. The Intel DPDK driver configures the device differently: no interrupts are generated and the driver does not read device registers to determine if packets have been received/transmitted ${ }^{6}$. The graphs for the optimized NIC show that even a moderate amount of optimization on both the device and the driver side can significantly improve the achievable throughput.

Our PCIe model is not limited to calculating achievable throughput for NICs. It can equally be used to programmatically model any PCIe device, provided that some details, typically obtainable from the device driver or the data sheet, are available. Furthermore, the model can and has been used to quickly assess the impact of alternatives when designing custom NIC functionality. The model has some limitations: Some of the lower level PCIe overheads, in particular for flow control messages are only estimated based on the PCIe specification and the model slightly overestimates their impact. Furthermore, the model does not account for PCIe overheads of unaligned DMA reads. For these, the specification requires the first CplD to align the remaining CplDs to an advertised Read Completion Boundary (RCB, typically 64B) and unaligned PCIe reads may generate additional TLPs.

\section{THE PCIE-BENCH METHODOLOGY}

While the PCIe protocol stack is relatively easy to model, it is the implementation of the DMA engine and, in particular, the increased complexity of the PCIe root complex, discussed in $\S 2$, which makes it hard to evaluate the end to end PCIe performance in real systems. Therefore, we designed a set of PCIe micro-benchmarks in the spirit of lmbench [39] and hbench:OS [5]. The main idea is to perform individual PCIe operations from a device to a buffer in host memory while carefully controlling the parameters which may affect performance.

Figure 3 illustrates the host buffer setup and the parameters which determine the access from a PCIe device. We define a (logically) contiguous buffer on the host. The buffer may be contiguous in DMA address space or comprised of a number of smaller buffers. It must be significantly larger than the size of the Last Level Cache (LLC), because, on some architectures, the PCIe root complex interfaces with the cache

\footnotetext{
${ }^{6}$ The DPDK driver polls on descriptor queues in host memory instead, checking for the valid fields indicating that a descriptor has been updated.
}

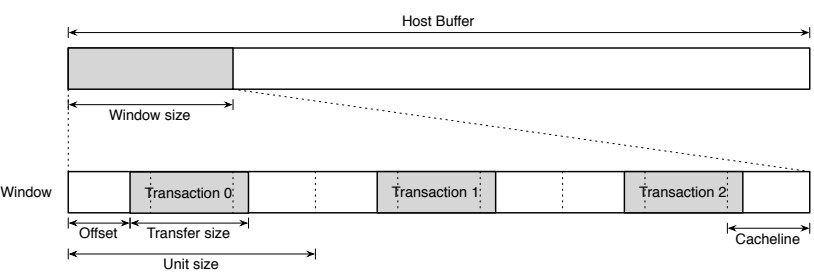

Figure 3: Host buffer layout and access parameters

system of the CPUs. To measure cache effects, only a subset of the host buffer, the window size, is accessed repeatedly.

For a given micro-benchmark we access the window size of the host buffer with multiple DMA requests and keep the amount of data transferred with each request fixed (transfer size). A DMA may start at an offset from a host cache line, allowing us to determine if there are any penalties for unaligned accesses. To ensure that each DMA request touches the same number of cache lines, the window is divided into equal sized units. Each unit is the sum of offset and transfer size, rounded up to the next cache line. A micro-benchmark can typically be configured to access the units sequentially or randomly (access pattern), though for most experiments in this paper we use random access. For each benchmark, the state of the CPU cache needs to be carefully controlled. By default, the cache is thrashed before every benchmark to ensure a cold cache. Optionally, we may attempt to warm the cache from the host, by writing to the window (host warm), or from the device. The latter is achieved by issuing a number of write DMAs to the window (device warm), before running tests. Understanding the impact of the host cache system is important: when transmitting packets from the host, typically, at least the packet header would be cache resident, and when receiving from the network, the packet data may get DMAed to non-cache resident buffers, depending on the overall pressure on the caches. Finally, the locality of the host buffer needs to be controlled. In SMP systems with integrated PCIe and memory controller, the entire host buffer is either allocated local to the node the PCIe device is attached to, or to a different (non-local) node.

\subsection{Latency benchmarks}

The first set of the PCIe micro-benchmarks measures the latency of individual PCIe operations. Measuring the latency of a PCIe Memory Read (MRd) transactions (from the device) is relatively easy on most programmable hardware: take a timestamp before issuing a DMA Read and another timestamp when the completion of the DMA Read is signaled by the DMA engine, then log the time-difference. DMA Read latency benchmarks are labeled LAT_RD throughout. 
The latency of DMA Memory Writes can not be measured directly from the device as PCIe Memory Write (MWr) transactions are posted: they are sent without explicit acknowledgment of success. Instead, we indirectly measure the latency of writes by issuing a DMA Write followed by a DMA Read from the same address. PCIe ordering ensures that the PCIe root complex handles the Read after the Write. Note, this approach does not allow us to compute the latency of DMA Writes alone as the latency of the DMA Read may be affected by the preceding DMA Write. The results from these benchmarks are labeled LAT_WRRD. For latency benchmarks we record the latency for each transaction and then calculate the average, median, min, max, 95th, and 99th percentile.

Latency measurements allow us to assess the cost of cache and IO-TLB misses, or the added latency for accessing remote memory from a PCIe device. While we can not measure the latency of a DMA Write directly, the latency of a DMA Write followed by a DMA Read to the same address provides insights into technologies, such as DDIO. Measuring latency includes the device overheads, such as the cost of issuing DMA requests or receiving notifications of completions. Therefore, when looking at the impact of the PCIe root complex, it is more insightful to study the relative change in latencies rather than the absolute latency values.

As highlighted in Section 2, latency measurements are of particular importance when writing software for programmable NICs or when implementing DMA engines in (reconfigurable) logic: the measured latency in Figure 2 by far exceeds the inter-packet times for small packets. Measured latency and its variance determines how many in-flight DMAs need to be handled.

\subsection{Bandwidth benchmarks}

The second set of PCIe micro-benchmarks focuses on bandwidth. DMA bandwidth is measured by taking a timestamp at the start, issuing a significant number of DMAs and taking a timestamp at the end. The bandwidth can be calculated by the time difference and the amount of data transferred. We are interested in straight DMA Read and DMA Write bandwidth (labeled BW_RD and BW_WR respectively). To measure bi-directional bandwidth we issue alternating DMA Read and Write transactions (labeled BW_RDWR). This ensures that PCIe MRd TLPs compete with PCIe MWr TLPs for the bandwidth to the root complex.

Bandwidth benchmarks, especially with small transfer sizes and random access patterns, generate a significant load on the system. If the DMA engine can saturate the PCIe link with 64 byte transfers, this would generate around 69.5 million transactions per second in each direction with the root complex handling a transaction every $5 n s$. Therefore, bandwidth micro-benchmarks can expose limitations in the root complex implementation as well as stressing the implementation of a device's DMA engines.

\section{IMPLEMENTATIONS}

The pcie-bench methodology requires programmatic and fine grained control over the PCIe device's DMA engines. We have implemented the methodology on both commercial boards from Netronome and research oriented NetFPGA boards. Two independent implementations of the same methodology validate our performance measurements against different host architectures and provide direct insights into two different PCIe implementations.

\subsection{Netronome NFP implementations}

Netronome offers a number of highly programmable NICs based on the NFP-6000 and NFP-4000 Ethernet controllers [44, 45, 59]. These Ethernet controllers feature up to 120 eight-way multi-threaded Flow Processing Cores (FPCs), a hierarchical memory subsystem, and fully programmable PCIe interfaces, all interconnected via a distributed switch fabric. The PCIe subsystem exposes two interfaces to the FPCs: A command interface allowing the core to issue small PCIe read and write transactions directly from registers and a bulk DMA interface allowing FPCs to en-queue large transfers between host memory and memory on the NFP. The PCIe micro-benchmarks are implemented as firmware on the FPCs utilizing both interfaces. The micro-benchmarks work both on NFP-4000 and NFP-6000 based controllers.

Firmware. The full PCIe micro-benchmark suite is implemented in a single firmware image. It provides a simple control interface, allowing a user space program on the host to select which type of benchmark to run and provide the necessary configuration parameters, such as the host buffer location, transfer size etc. Benchmark results are written to NFP memory where they can be read back from the host.

Latency tests are run on a single thread in one of the FPCs. This thread calculates the next host address to use and prepares a DMA descriptor. The thread then reads the current value of the internal timestamp counter and enqueues the DMA descriptor to the DMA engine. The DMA engine signals the thread once the DMA completed and the thread takes another timestamp and journals the difference to memory on the NFP. A variant of the latency benchmarks uses the direct PCIe command interface, which is suitable for small transfer (up to 128 bytes). With this variant, instead of building and enqueuing a DMA descriptor, the thread can directly issue the PCIe read or write commands and gets signaled on completion. The timestamp counter increments every 16 clock cycles, which, on a $1.2 \mathrm{GHz}$ NFP, provides a resolution of $19.2 n s$. For latency benchmarks we typically journal the timing for 2 million transactions. 
For DMA bandwidth tests, the challenge is to ensure that DMA descriptors are enqueued to the DMA engine at a higher rate than it drains the queue. To achieve this for small transfer sizes, we use 12 cores with 8 threads as DMA workers. In a loop, each DMA worker thread calculates a host address, prepares and enqueues a DMA descriptor and yields until the completion is signaled. An atomic counter, shared between all workers, is decremented before issuing a new DMA request. If the counter value goes below zero, the worker stops. For BW_RDWR tests, each worker issues a DMA Read if the counter is even and a DMA Write when the counter is odd. A control thread is used to start all the worker threads and waits for them to complete. The elapsed time is used to calculate the achieved bandwidth. For bandwidth tests 8 million DMA requests are performed. For both DMA latency and bandwidth tests the DMA descriptors are configured to target an internal 256 KB SRAM, known as the Cluster Target Memory. This memory has an access latency of 50-100 cycles. Enqueuing DMA descriptors incurs a similar latency. These latencies add a fixed cost to each DMA transfer, and while they are specific to the NFP, we expect other programmable NICs to have similar overheads. The firmware is implemented in Micro-C, an NFP specific extension to $\mathrm{C}$. The micro-benchmark suite is implemented with around 1500 lines of code, with the core requiring around 500 lines of code. The code can be compiled, without external dependencies, to run on NFP6000 and NFP4000 based networking cards.

\subsection{NetFPGA}

NetFPGA is an open-source community platform [43]. It is supported by cutting-edge reconfigurable boards, reference software and hardware designs, as well as the infrastructure for design development and verification. The latest generation of NetFPGA board, NetFPGA-SUME, is a PCIe host adapter card [62] capable of $100 \mathrm{~Gb} / \mathrm{s}$ application speeds. The board utilizes a large Xilinx Virtex-7 FPGA device incorporating two PCIe Gen 3 hard-blocks, along with resources such as QDRII+ and DDR3 memory types.

Hardware. The PCIe micro-benchmark suite is implemented directly on the FPGA. It enhances the DMA engine described in [61] with pcie-bench capabilities. The system provides a simple control interface, allowing the host to select which micro-benchmark to run along with its parameters. The system keeps track of time through a free-running counter, operating at PCIe core frequency $(250 \mathrm{MHz})$, providing a resolution of $4 n s$. Every time the software triggers a new microbenchmark, a finite state machine coded into the hardware, calculates the host addresses and generates the associated memory read or write request. The design does not use a FIFO to enqueue DMA requests, instead the DMA requests are directly passed to the DMA engine. All the memory requests are generated on-the-fly as the hardware design allows transmission of a new request every clock cycle.

For latency tests, the system is configured to take a timestamp before a DMA read and after the acknowledgment signal is received. The system records up to 1000 latency values. For bandwidth tests, the system measures the total time it takes to perform 1 million transactions. Benchmark results are written to NetFPGA memory after a benchmark run, where they can be read back from the host. The FPGA design is written in Verilog and System Verilog. The microbenchmark suite is implemented with around 1200 lines of code. It can be compiled for NetFPGA-SUME and Xilinx VC709 boards.

\subsection{Kernel drivers}

Both the NFP and the NetFPGA implementations use a kernel driver to initialize the hardware, allocate host memory for DMAs and provide access to user space programs to control the execution of the benchmarks and to collect the results. The NFP pcie-bench driver uses the standard NFP kernel driver $^{7}$. It allocates the host side DMA buffers in chunks of $4 \mathrm{MB}$ as this the maximum size which can be allocated physically contiguous on most Linux kernel versions. The NetFPGA driver allocates memory either from hugetlbfs (with $1 \mathrm{~GB}$ pages) or standard $4 \mathrm{~KB}$ system pages. huget lbfs is the default option as it allows for the easy allocation of large, physically contiguous areas of memory. Both drivers provide control over which NUMA node memory is allocated from and export an interface allowing a user space program to warm the caches with the controlled portions of the host DMA buffers. The NFP pcie-bench kernel module is implemented in around 400 lines of code while the NetFPGA driver is implemented in approximately 800 lines of code.

\subsection{Control programs}

The execution of benchmarks, gathering of the data and postprocessing of the results is performed by user space programs in both implementations. The NFP implementation uses a Python program with a small utility, written in $\mathrm{C}$, to handle cache warming. The Python program can be used to run individual tests or a full suite of tests. A complete run takes about 4 hours and executes around 2500 individual tests. For latency benchmarks, the control program reads the timing data of the individual transactions and calculates various metrics, such as the average, median, min, max and 95th and 99th percentile. Optionally, it generates CDFs, histograms and time series of the data. For bandwidth tests, the control program calculates the bandwidth and transaction rate. It is implemented in 1600 lines of Python and 120 lines of $\mathrm{C}$ code.

\footnotetext{
${ }^{7}$ https://github.com/Netronome/nfp-drv-kmods
} 
The NetFPGA control program is implemented in approximately 600 lines of $C$ code and provides a command line interface to control the individual test parameters. The raw test results are written to a file for further processing.

\subsection{Implementation on other devices}

pcie-bench is best implemented on PCIe devices providing programmatic, fine-grained control over the DMA engines, such as the Netronome NFP and NetFPGA described above. There is a growing number of PCIe cards with similar capabilities. For example, the Intel Xeon Phi coprocessor exposes the device's DMA engines via descriptor rings both to the Phi's cores and the host [18]. There are also a growing number of programmable NICs with integrated FPGAs, such as the already mentioned Exablaze NICs [11] and Mellanox's Programmable ConnectX-3 Pro [40]. Implementing pcie-bench on these devices should require roughly the same effort as it took for the initial two implementations.

Some limited aspects of pcie-bench could potentially be implemented on non-programmable, commodity NICs in loopback mode by carefully controlling the locations of buffers used for packet send and receive. For example, one could repeatedly enqueue the same packet buffer for transmit and vary the freelist buffers to direct received packets to a variable window size (or vice versa). The relative changes in latency or bandwidth may provide some insight into host side PCIe implementation and changing the alignment of buffer addresses may reveal limitations of a device's DMA engines. Note, however, the results will likely be less accurate than those obtainable with programmable PCIe devices as measurements with commodity NICs would always include overheads for descriptor transfers.

\section{EXPERIMENTAL EVALUATION}

This section reports the pcie-bench suite in action. Table 1 lists the details of the systems used for the evaluation results presented in this paper. We focus on systems built around Intel's Xeon E5 processors as this is the most common configuration used in datacenter servers at the time of writing [55]. We discuss results obtained comparing several generations of Xeon E5 processors but also include some data from an Intel Xeon E3 system to compare results with a different processor architecture. For all experiments we use a PCIe Gen 3 $\mathrm{x} 8$ configuration as it is the default for modern NICs. We expect the pcie-bench methodology to be equally applicable to other PCIe configurations including the next generation PCIe Gen 4 once hardware is available.

\subsection{Baseline bandwidth and latency}

As the first set of results we present the baseline throughput of the NFP-6000 and NetFPGA implementations in Figure 4.
We compare them to the required bandwidth for $40 \mathrm{~Gb} / \mathrm{s}$ Ethernet as well as a simplified model of achievable throughput for a PCIe Gen 3 device. The model accurately calculates the overhead of the physical, data link, and transaction layer of PCIe but only estimates the overhead of flow control messages. We measure the throughput of PCIe read, write, and alternating read/write transfers for different transfer sizes to a fixed, $8 \mathrm{~KB}$ host buffer, which is warmed before each test to eliminate any caching effects. We vary the transfer size from $64 \mathrm{~B}$ to $2048 \mathrm{~B}$ in rough increments of powers of 2 but take additional measurements with $-1 /+1 B$ around important transfer sizes, such as some cache line or TLP size boundaries. All DMA start addresses are cache line aligned and all tests were performed on the same Xeon E5 2637v3 system to eliminate any differences in system configuration. Figure 4 shows the results.

In all three data sets, the NetFPGA implementation of pcie-bench closely follows the PCIe bandwidth calculated with our model. For PCIe writes the NetFPGA implementation achieves a slightly higher throughput though. This is because the model assumes a fixed overhead for flow control messages which, for uni-directional traffic, would not impact traffic throughput. The NFP implementation of pcie-bench generally achieves slightly lower throughput than the NetFPGA implementation (but typically achieves throughput sufficient to support $40 \mathrm{~Gb} / \mathrm{s}$ Ethernet rates). The main reason is that the NetFPGA implementation directly drives the DMA engines from the internal FPGA memory and does not perform any additional processing. In the NFP implementation, the DMA engines must also transfer data to/from the host into internal SRAM, and then transfer it further into NFP internal memory where it can be accessed by the FPCs of the NFP card. These additional overheads, as well as the required buffer management and signaling, introduce a slight performance degradation. Finally, it is worth noting that each graph shows that neither implementation is able to achieve a read throughput required to transfer $40 \mathrm{~Gb} / \mathrm{s}$ Ethernet at line rate for small packet sizes.

Next, we look at the latency of individual DMA transactions varying the transfer size (Figure 5). The setup is the same as for the bandwidth results described above. Overall, the latency numbers for both the NFP-6000 and NetFPGA are of the same order of magnitude, indicating that the bulk of the latency can be attributed to general PCIe and overall host overheads. It is very similar across the four generations of Intel processors we measured and is also in line with the latency measured using the ExaNIC presented in $\S 2$.

The latency for DMA requests is higher on the NFP-6000 with an initial fixed offset of about $100 \mathrm{~ns}$ for smaller transfers, and the gap increasing for larger transfers. The reasons are twofold. The fixed offset can be explained with the overhead of enqueuing DMA descriptors to the DMA engines, 


\begin{tabular}{|c|c|c|c|c|c|c|}
\hline Name & $\mathrm{CPU}$ & NUMA & Architecture & Memory & OS/Kernel & Network Adapter \\
\hline NFP6000-BDW & Intel Xeon E5-2630v4 2.2GHz & 2-way & Broadwell & $128 \mathrm{~GB}$ & Ubuntu 3.19.0-69 & NFP6000 1.2GHz \\
\hline NetFPGA-HSW & \multirow{2}{*}{ Intel Xeon E5-2637v3 3.5GHz } & \multirow{2}{*}{ no } & \multirow{2}{*}{ Haswell } & \multirow{2}{*}{ 64GB } & \multirow{2}{*}{ Ubuntu 3.19.0-43 } & NetFPGA-SUME \\
\hline NFP6000-HSW & & & & & & NFP6000 1.2GHz \\
\hline NFP6000-HSW-E3 & Intel Xeon E3-1226v3 3.3GHz & no & Haswell & $16 \mathrm{~GB}$ & Ubuntu 4.4.0-31 & NFP6000 1.2GHz \\
\hline NFP6000-IB & Intel Xeon E5-2620v2 2.1GHz & 2-way & Ivy Bridge & $32 \mathrm{~GB}$ & Ubuntu 3.19.0-30 & NFP6000 1.2GHz \\
\hline NFP6000-SNB & Intel Xeon E5-2630 2.3GHz & no & Sandy Bridge & $16 \mathrm{~GB}$ & Ubuntu 3.19.0-30 & NFP6000 1.2GHz \\
\hline
\end{tabular}

Table 1: System configurations. All systems have 15MB of LLC, except NFP6000-BDW, which has a 25MB LLC.

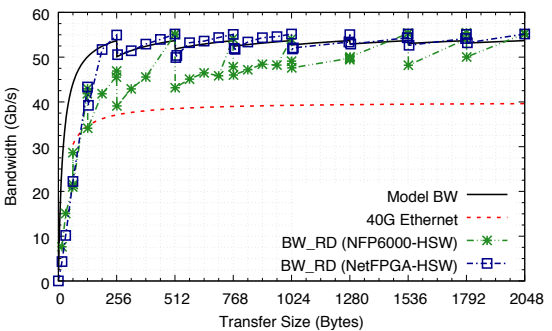

(a) PCIe Read Bandwidth

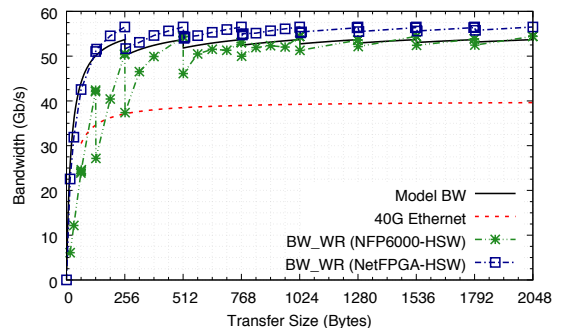

(b) PCIe Write Bandwidth

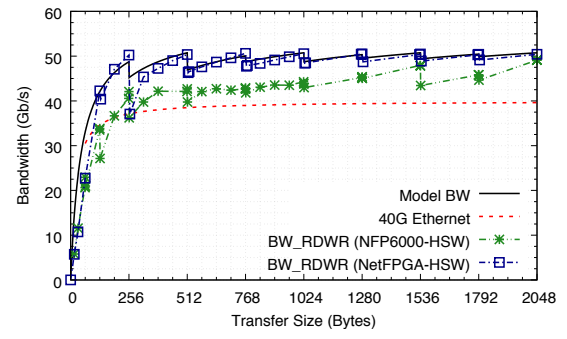

(c) PCIe Read/Write Bandwidth

Figure 4: Baseline PCIe DMA bandwidth for NFP6000-HSW and NetFPGA-HSW with warm caches.

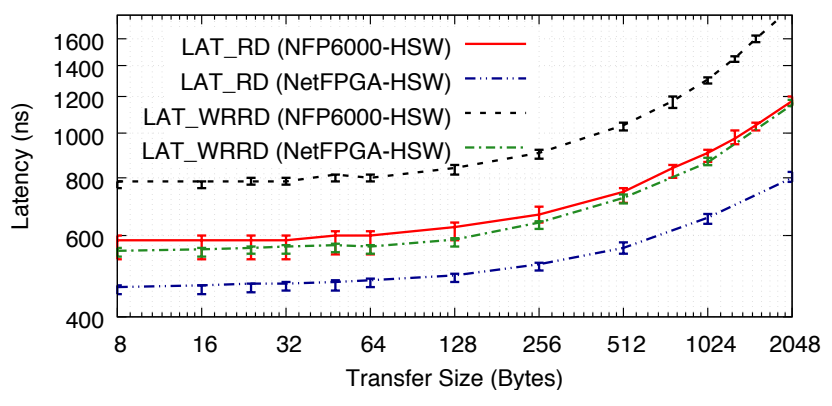

Figure 5: Median DMA latency for NFP-6000 and NetFPGA; minimum and 95th percentile are shown as error bars.

which is avoided in the NetFPGA implementation. When using the NFP's direct PCIe command interface, designed for smaller transfers, the NFP-6000 achieves the same latency as the NetFPGA, further indicating that the majority of the latency can be attributed to host system. The widening of the gap as the transfer size increases can be explained by considering the internal architecture of the NFP (§ 5.1). Every DMA transfer to/from the host causes an additional transfer from the internal SRAM to NFP internal memory. This additional transfer increases the latency depending on the transfer size, but it is useful for decoupling the DMA request from the (variable) packet processing times performed by the FPCs.

\subsection{Comparing architectures}

Figure 5 shows that the minimum and 95th percentile of DMA latencies for a Intel Xeon E5 system are very close to the median latency, suggesting that there is little variance in the latency experienced by PCIe transactions. Figure 6 shows the distribution of 64B DMA read latencies for the same system (NFP6000-NFP) as a CDF. The graph confirms that $99.9 \%$ of all transactions fall inside a narrow 80ns range starting with a minimum of $520 \mathrm{~ns}$ and a median of $547 \mathrm{~ns}$. The maximum latency out of 2 million transactions was $947 n$ s.

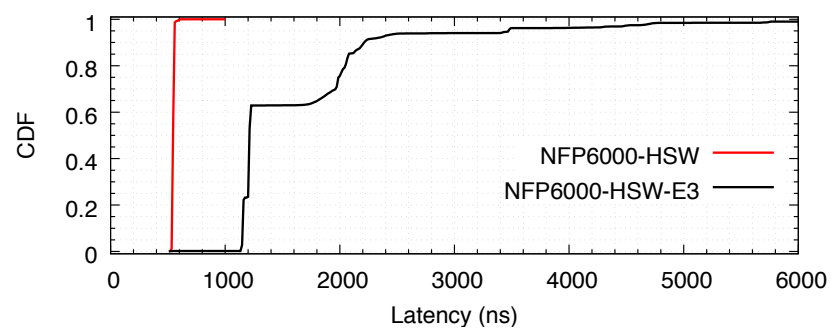

Figure 6: Latency distribution for Xeon E5 and E3 for 64B DMA reads with warm caches.

The figure also shows the result from a Xeon E3 system of the same processor generation (NFP6000-HSW-E3). The results stand in stark contrast to those from the Xeon E5 system. The minimum latency on the Xeon E3 system is actually lower with $493 n s$ but the median is more than double 
with $1213 n s$. Furthermore, from around the 63rd percentile the latency increases dramatically with the 90th percentile being double the median and the 99th percentile being $5707 n s$. The 99.9th percentile (11987ns) is an order of magnitude larger than the median with most latencies beyond being larger than $1 \mathrm{~ms}$ up to a maximum latency of $5.8 \mathrm{~ms}$.

The differences are also reflected in the bandwidth benchmarks (not shown) where for DMA reads the Xeon E3 system only matches the Xeon E5 system for transfers larger than 512B and, for DMA writes, never achieves the throughput required for $40 \mathrm{~Gb} / \mathrm{s}$ Ethernet for any transfer size.

We can only speculate on the causes for this different behavior. It seems likely that Intel maintains completely different PCIe root complex implementations for its Xeon E5 and Xeon E3 lines of processors. Looking in more detail at the Xeon E3 data, there is no regular pattern to the occurrence of the longer latencies. We suspect that, in particular the larger latencies may be related to hidden power saving modes. We have previously observed large $(<1 \mathrm{~ms})$ latencies on Xeon E5 systems before disabling power saving modes in the BIOS. However, disabling them on the Xeon E3 system did not significantly change the latency distribution.

\subsection{Caching and DDIO}

We now examine the impact of caching and DDIO on PCIe transactions. For these experiments we keep all parameters constant, except for the window size and the state of the LLC. The window size was varied from $4 \mathrm{~KB}$ to $64 \mathrm{MB}$, exceeding the $15 \mathrm{MB}$ or $25 \mathrm{MB}$ size of the LLC on all our systems.

Firstly, we look at PCIe transaction latency. To measure the latency we use the NFP's PCIe command interface with 8 Byte transactions, as it is the lowest latency operation we have available. We ensure that each subsequent transaction touches a different cache line as described in Section 4.1. The results are shown in Figure 7(a). For PCIe reads (LAT_RD) with a cold cache we see no changes in latency as we change the window size: All PCIe reads are serviced from memory. When the cache is warm, the read latency is around 70ns lower but increases once the window size exceeds the size of the LLC. This confirms that PCIe reads are serviced from the LLC if the data is resident in the cache. With a warm cache, the latency of a posted PCIe write followed by a read (LAT_WRRD) follows roughly the same pattern: writes and reads hit the LLC and, once the window size exceeds the LLC size, the latency increases by around 70ns. For a cold cache the LAT_WRRD results illustrate the effect of DDIO: For small window sizes new cache lines get allocated and writes (and subsequent reads) are performed to/from the cache. Once the window size exceeds the $10 \%$ of the LLC reserved for DDIO, dirty cache lines have to be flushed out to memory before the write can succeed, causing a 70ns delay for most writes.
For larger transfer sizes, the differences between hitting the cache or not is reduced significantly: for 64B LAT_WRRD tests the difference is around 10ns.

The bandwidth data, presented in Figure 7(b), suggest a similar story. For 64B DMA Reads (BW_RD), there is a measurable benefit if the data is already resident in the LLC. For larger transfer sizes (not shown) the benefit is smaller, and from 512B DMA Reads onwards, there is no measurable difference. For DMA Writes (BW_WR), there is no benefit if the data is resident in the cache or not. There is also no measurable benefit on keeping the windows size below the $10 \%$ of the LLC. We suspect that the DDIO portion of the cache is cleaned quick enough, so that all DMA Writes either hit the main LLC or the DDIO portion. Since DDIO can not be disabled and there are no dedicated performance counters it is not possible to validate this hypothesis.

\subsection{NUMA impact}

With the PCIe root complex and memory controllers integrated in each CPU node, a PCIe device may access memory local or remote to the node to which it is attached. In this section we evaluate the performance impact of this nonuniform memory access. The host buffer is allocated either on the node the PCIe device is attached to (local) or on the other node in a 2-way NUMA system (remote). The cache is warmed or thrashed on the node the buffer is allocated from.

Figure 8 shows the percentage change for DMA read bandwidth of local memory versus remote memory for different transfer sizes across different window sizes with a warm cache. 64B DMA reads experience a 20\% drop in throughput (from $\sim 32 \mathrm{~Gb} / \mathrm{s}$ to $\sim 25 \mathrm{~Gb} / \mathrm{s}$ ). The difference drops to around $10 \%$ once the DMAs are not serviced from the local cache anymore. With 128 and 256B DMA reads the penalty for accessing remote memory drops to $5-7 \%$ (e.g., from $\sim 44 \mathrm{~Gb} / \mathrm{s}$ to $\sim 41 \mathrm{~Gb} / \mathrm{s}$ for $128 \mathrm{~B}$ ). There is no noticeable penalty for $512 \mathrm{~B}$ DMA reads. The data is confirmed by cold cache DMA read throughput (not shown) where remote 64B reads experience a constant $10 \%$ penalty ( $\sim 5 \%$ for $128 \mathrm{~B}$ and $256 \mathrm{~B}$ reads).

The throughput of DMA Writes does not seem to be affected by the locality of the host buffer nor by the size of the host buffer. In contrast with the Intel specification [20], we believe that all DMA Writes may be initially handled by the local DDIO cache.

For completeness, the latency numbers do not differ much from the local case presented in Figures 5 and 7 except that remote accesses add a constant $100 \mathrm{~ns}$ of latency to our systems. Overall, the results from our two dual socket systems (NFP6000_BDW and NFP6000_IB) are the same, indicating there has been little change in the two generations separating them. 


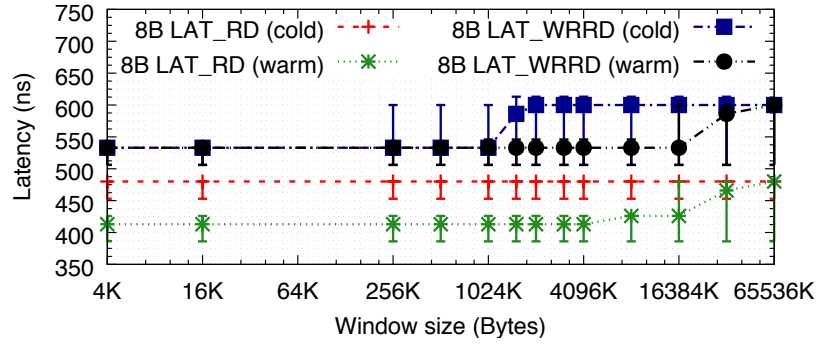

(a) Median latency; minimum and 95th percentile are shown as error bars.

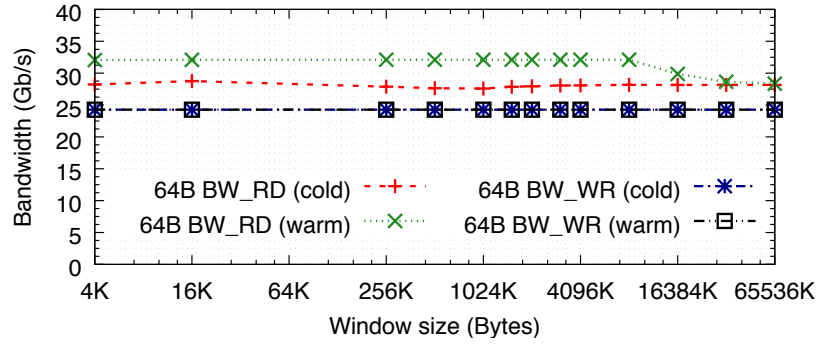

(b) Bandwidth

Figure 7: Cache effects on Latency and Bandwidth (NFP6000-SNB)

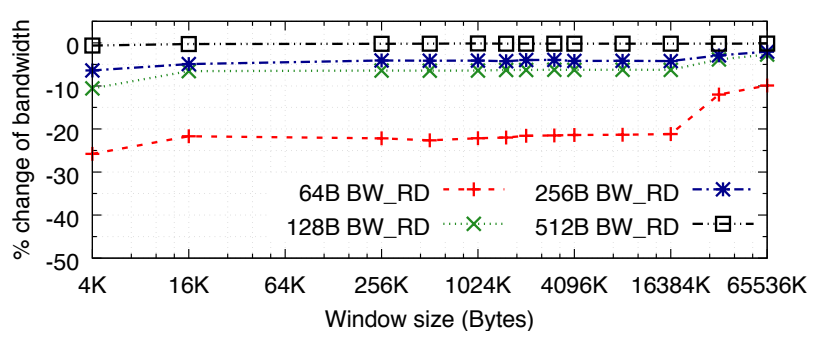

Figure 8: Difference between local and remote DMA Reads of different sizes with warm cache (NFP6000-BDW).

\subsection{The IOMMU effect}

Finally, we look at the impact of an IOMMU interposed in the datapath between PCIe devices and the host. To measure the impact of the IOMMU, we enable it on the Linux kernel command line with intel_iommu=on. IOMMU implementations in Ivy Bridge and newer architectures support super-pages to reduce the number of page table entries, and thus reduce the number of TLB entries. For our experiments we disable this behavior (by also specifying sp_off on the kernel command line). This forces the use of $4 \mathrm{~KB}$ page table entries and allows us to use a relatively small 64MB host buffer for our experiments.

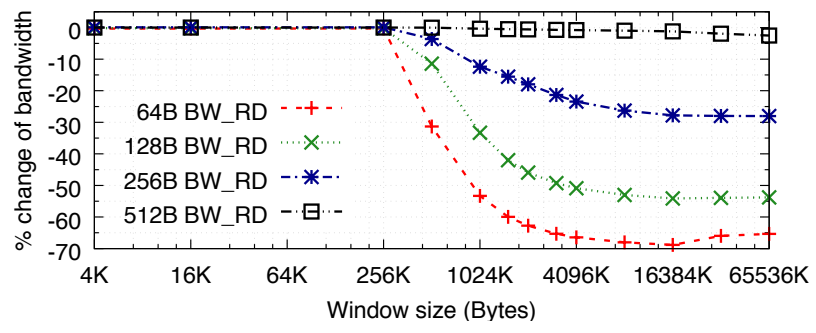

Figure 9: Impact of IOMMUs on DMA reads of different transfer sizes with warm caches (NFP6000-BDW).
Figure 9 shows the result for different transfer sizes. Like the data presented in Section 6.4 the graph shows the percentage change to the same experiment run without IOMMU. For small window sizes, there are no measurable differences across the range of transfer sizes. However, once the window size is increased beyond $256 \mathrm{~KB}$, the throughput drops dramatically. For 64B DMA Reads it drops by almost $70 \%$ and even for 256B DMAs the drop is still a significant $30 \%$. There is no change for transfer sizes of $512 \mathrm{~B}$ and above. For DMA Writes (not shown), the drop is not quite as dramatic $(55 \%$ for $64 \mathrm{~B}$ writes), but is still very significant.

From this data we conclude that on that particular system the IO-TLB has 64 entries $(256 K B / 4 K B)$, a number that is not published by Intel. The latency of 64B Reads increases from around $430 n s$ to $760 n s$, putting the cost of an IO-TLB miss and subsequent page table walk at around 330ns. For smaller transfer sizes this penalty is relatively high compared to the time it takes to transfer the data, and thus the impact on the throughput is higher still. The data is surprisingly consistent across all 4 generations of Intel micro-architectures where we ran the experiments, and we observe the same effects with our NetFPGA pcie-bench implementation. With such consistent results across the 4 micro-architecture generations we conclude Intel's IOMMUs have undergone little development since their first implementation.

\section{LESSON LEARNED AND USE CASES}

The results obtained from pcie-bench can be and have been used to characterize and tune system software for high performance I/O. There are a plethora of sophisticated tools to analyze operating system and application performance, e.g., $[14,21]$, as well as tools to understand the performance impact of host CPU architectures and OS primitives, e.g., $[5,39]$. However, none of these tools provide detailed insights into the performance of the I/O subsystem. pcie-bench provides the necessary data to fine-tune specialized network stacks [37, 38], and optimize kernel IOMMU handling [48]. 


\begin{tabular}{|l|l|l|}
\hline Area & Observation & Evaluation-directed recommendation \\
\hline \hline IOMMU (§6.5) & Significant throughput drops as working-set size increases. & Co-locate I/O buffers into superpages. \\
\hline DDIO (§6.3) & Small transactions are faster when the data is resident in the cache. & $\begin{array}{l}\text { DDIO improves descriptor ring access and } \\
\text { performance for small packet receive. }\end{array}$ \\
\hline $\begin{array}{l}\text { NUMA (§6.4) } \\
\text { (small transactions) }\end{array}$ & Higher cost of DMA reads from remote memory compared to local caches. & Place descriptor rings on the local node. \\
\hline $\begin{array}{l}\text { NUMA (§6.4) } \\
\text { (large transactions) }\end{array}$ & No significant difference between remote and local cache performance. & $\begin{array}{l}\text { Place packet buffers on the node where } \\
\text { processing happens. }\end{array}$ \\
\hline
\end{tabular}

Table 2: Notable findings from this paper, derived experimentally with pcie-bench.

Table 2 reports the notable findings from this paper which have been derived experimentally with pcie-bench. Based on the IOMMU data, we strongly recommend using superpages and trying to force the IO buffers used for DMA to be co-located in as few super-pages as possible. This may be possible in carefully controlled environments, such as virtualized network appliances. However, in multi-tenant Virtual Machine (VM) environments offering assigned PCIe devices to VMs, it is currently not possible to isolate the IO performance of VMs sufficiently with Intel's IOMMUs.

For NUMA architectures, we found that small DMA reads from the remote cache are significantly more expensive than reads from the local cache. Accessing data on a remote node also adds around 100ns of latency. While it may not be feasible to "pin" small network packets to the local node, it would certainly be useful to locate data structures, such as descriptor rings, on the local node. Our data suggests that, for larger packet sizes, the locality of the packet buffer is not critical and it is recommended to allocate data on the nodes where the processing happens.

Finally, our measurements confirm the documented operation of DDIO and show that, for small transfers, accesses are around $70 \mathrm{~ns}$ faster for cache resident data. We see two areas where this can be beneficial for network applications. Firstly, access to descriptor rings is lower latency, and therefore incur less overhead. Secondly, the integration with the caches should benefit small packet receive, in particular for packet sizes which are not multiples of a cacheline (e.g., 64B Ethernet frames with the 4B FCS stripped). Since the data is directly DMAed into the cache, dirty cachelines do not need to be written back to memory before the DMA can succeed.

While the above lessons focus on optimizing host system software, the insights gained with pcie-bench have also implications for the growing area of research moving beyond straightforward TCP offload to offloading more complex processing to programmable and reconfigurable network cards, e.g., $[2,26,51,52]$. In order to implement the firmware or reconfigurable logic for these application-offloads, it is important to have a detailed characterization of the DMA engines and their interaction with the host system. The data provided by pcie-bench is ideal to guide such implementation choices. For example, understanding the latency of transactions for the Netronome boards has heavily influenced the design of multiple firmware implementations that offer different offloads of network processing. The latency data determines how many in-flight DMAs the firmware has to handle (for both packet data DMA and descriptor DMAs) to sustain line rate. In turn, this determines the sizing of I/O structures such as rings and other storage for in-flight DMAs along with specifying the appropriate number of Flow Processing Cores and threads. The latency and bandwidth data also determines the amount of batching performed both in the firmware and the corresponding device driver in the operating system. For example, on the NFP6000-HSW system, it takes between $560-666 n$ s to transfer $128 \mathrm{~B}$ of data from the host to the device. At $40 \mathrm{~Gb} / \mathrm{s}$ line rate for $128 \mathrm{~B}$ packets, a new packet needs to be transmitted every 29.6ns. This means that the firmware and DMA engines need to handle at least 30 transactions in flight. These calculations can be extended to take into account the latency for descriptor transfers and to work out the cycle budget for each DMA. If the IOMMU is enabled, the firmware and DMA engines also need to cover the occasional latency increase of $\sim 330 \mathrm{~ns}$, caused by IOMMU TLB misses. Furthermore, we have seen significant variance in latencies on some systems, most notably on a Xeon E3 system, which further complicate the implementation of high performance firmware or DMA engine designs.

Finally, the pcie-bench methodology and measurements can be used by silicon engineers to evaluate existing designs and inform the objectives of future architecture of DMA engines. For example, the PCIe-latency measurements were used to assess each iteration of a suite of NetFPGA DMAengine designs. Being able to compare the current design with data from other implementations helps to determine if a performance bottleneck is due to artifacts in the host architecture or is a design limitation. More importantly, the methodology was also extensively used for validation during chip bring-up, and to guide architectural decisions in future silicon at Netronome. Using micro-benchmarks, as provided by pcie-bench, are ideal as they provided detailed and controlled data which is not obtainable by other means. 


\section{RELATED WORK}

Several papers have observed PCIe and its interactions with host architecture in the context of higher performance network applications. Kalia et al. [24] provide a low level evaluation and recommendation for RDMA primitives and how they interact with PCIe. Li et al. [32] discuss the benefits of DDIO on the performance of modern Key-Value-Store (KVS) applications. The impact of NUMA on device DMA was discussed by Lim et al. [34] in relation to high performance KVS applications and by Han et al. [17] for GPU accelerated packet processing. All these touch on some aspects covered in this paper but in the context of some higher level applications. In contrast, pcie-bench allows for a systematic study of PCIe performance characteristics. The results obtained can then be used to explain application performance behavior in detail and may guide future application level performance improvements more accurately.

As for more generic studies about PCIe, there has been little micro-benchmark work beyond Miller et al. [41] who describes an approach to measure PCI and first generation PCIe interconnect latency through a differential analysis of transaction delays. Moll et al. [42] demonstrated the use of FPGA based hardware for profiling software applications including an analysis of PCI bus performance, while Endo et al. [10] demonstrate the importance of latency in interactive and transactional application software. In addition, they introduced a new set of micro-benchmarks to assess the performance against latency and encourage further, applicationspecific study of PCI interconnect latency impact.

Surveying specialist cases of PCIe, Lostrie et al. [36] present a setup for benchmarking the performance of the full PCIecommunication path between two FPGA-boards with microTCA. While, Koop et al. [27] evaluate InfiniBand on a PCIe 2.0 system. The authors study the benefits of PCIe 2.0 on both DDR and QDR data rates on the Mellanox ConnectX and also investigate the general trend of additional interconnect bandwidth upon application-performance on multi-core machines. Earlier still, Liu et al. [35] evaluate the third generation InfiniBand HCAs from Mellanox, which supports PCIe interfaces. They compare the performance of these with HCAs using PCI-X interfaces. The evaluation consists of a set of micro-benchmarks at the interconnect level, including latency, bandwidth, and bidirectional bandwidth experiments. They show that InfiniBand HCAs with PCIe interfaces deliver excellent performance. However, the methodology is only superficially similar to our own with a focus firmly on InfiniBand performance.

Finally, Braithwaite [4] and Li et al. [33] investigate I/O bandwidth in NUMA architectures. The former presents a method for analyzing main memory and PCIe data-access characteristics of modern AMD and Intel NUMA architectures. They also present the synthesis of data-access performance models designed to quantify the effects of these architectural characteristics on bandwidth. Li et al.further contribute a characterization of the state-of-the-art NUMA hosts, and propose a methodology to simulate I/O operations using memory semantics, and in-turn model the I/O bandwidth performance. In both cases, these efforts provide little insight into the explicit and evolving relationship between PCIe and modern NUMA architectures.

\section{CONCLUSION AND FUTURE WORK}

This paper shows that PCIe, alongside its interaction with the root complex and device drivers, can significantly impact the performance of end host networking. Past research has reported some of the findings in the context of specific applications, such as RDMA and KVS acceleration. In contrast, we provide a theoretical model and a methodology, pcie-bench, to understand and study inherent bottlenecks of the PCIe protocol. We also present two implementations of the methodology for a systematic evaluation and characterization of PCIe devices in real systems.

Beyond the pcie-bench design and implementation, we discuss our characterization results from a number of systems. We share lessons learned with implications for current software systems and future hardware designs. Our study allows exploration of the impact of new PCIe and host architecture features such as DDIO and IOMMUs. Specifically, we demonstrate that the PCIe integration with caches of DDIO works well but, also characterize the significant, and sometimes undesirable, impact that Intel IOMMUs and NUMA may have when high DMA rates are necessary.

Our initial results show that more work is to be done. Given the remarkable differences in PCIe performance between Xeon E5 and Xeon E3 systems, a more detailed study with different architectures, e.g., AMD, ARM64, and Power based servers, would provide further interesting insights into PCIe implementations. Furthermore, we have not yet studied the impact of multiple high performance PCIe devices in the same server, a common configuration in datacenters. Such a study would reveal further insights into the implementation of IOMMUs (e.g., are IO-TLB entries shared between devices) and potentially unearth further bottlenecks in the PCIe root complex implementation.

Acknowledgments. This research is (in part) supported by the UK's Engineering and Physical Sciences Research Council (EPSRC) under the EARL project (EP/P025374/1) and the European H2020 projects dReDBox (grant No. 687632) and METRO-HAUL (grant No. 761727). 


\section{REFERENCES}

[1] Hitesh Ballani, Paolo Costa, Christos Gkantsidis, Matthew P. Grosvenor, Thomas Karagiannis, Lazaros Koromilas, and Greg O'Shea. 2015. Enabling End-Host Network Functions. In Special Interest Group on Data Communication (SIGCOMM). ACM.

[2] Hitesh Ballani, Paolo Costa, Christos Gkantsidis, Matthew P. Grosvenor, Thomas Karagiannis, Lazaros Koromilas, and Greg O'Shea. 2015. Enabling End-Host Network Functions. In Special Interest Group on Data Communication (SIGCOMM). ACM.

[3] Nicola Bonelli, Andrea Di Pietro, Stefano Giordano, and Gregorio Procissi. 2012. On multi-gigabit packet capturing with multi-core commodity hardware. In Passive and Active Measurement (PAM). Springer.

[4] Ryan Karl Braithwaite. 2013. NUMA data-access bandwidth characterization and modeling. Master's thesis. Virginia Polytechnic Institute and State University, US.

[5] Aaron B. Brown and Margo I. Seltzer. 1997. Operating system benchmarking in the wake of Lmbench: a case study of the performance of NetBSD on the Intel x86 architecture. In Special Interest Group for the Computer Systems Performance Evaluation Community (SIGMETRICS). ACM.

[6] Martin Casado, Teemu Koponen, Scott Shenker, and Amin Tootoonchian. 2012. Fabric: A Retrospective on Evolving SDN. In Hot Topics in Software Defined Networks (HotSDN). ACM.

[7] Colin Dixon, Hardeep Uppal, Vjekoslav Brajkovic, Dane Brandon, Thomas Anderson, and Arvind Krishnamurthy. 2011. ETTM: A Scalable Fault Tolerant Network Manager. In Networked Systems Design and Implementation (NSDI). USENIX.

[8] DPDK. 2018. Official website. http://www.dpdk.org.

[9] Peter Druschel, Larry L. Peterson, and Bruce S. Davie. 1994. Experiences with a High-speed Network Adaptor: A Software Perspective. In Conference on Communications Architectures, Protocols and Applications (SIGCOMM). ACM

[10] Yasuhiro Endo, Zheng Wang, J. Bradley Chen, and Margo Seltzer. 1996. Using latency to evaluate interactive system performance. In Symposium on Operating Systems Design and Implementation (OSDI). ACM.

[11] Exablaze. 2018. Official website. http://www.exablaze.com.

[12] Daniel Firestone. 2018. Building Hardware-Accelerated Networks at Scale in the Cloud. https://conferences.sigcomm.org/sigcomm/2017/ files/program-kbnets/keynote-2.pdf.

[13] Daniel Firestone, Andrew Putnam, Sambhrama Mundkur, Derek Chiou, Alireza Dabagh, Mark Andrewartha, Hari Angepat, Vivek Bhanu, Adrian Caulfield, Eric Chung, Harish K. Chandrappa, Somesh Chaturmohta, Matt Humphrey, Jack Lavier, Norman Lam, Fengfen Liu, Kalin Ovtcharov, Jitu Padhye, Gautham Popuri, Shachar Raindel, Tejas Sapre, Mark Shaw, Gabriel Silva, Madhan Sivakumar, Nisheeth Srivastava, Anshuman Verma, Qasim Zuhair, Deepak Bansal, Doug Burger, Kushagra Vaid, David A. Maltz, and Albert Greenberg. 2018. Azure Accelerated Networking: SmartNICs in the Public Cloud. In Networked Systems Design and Implementation (NSDI). USENIX.

[14] Brandan Greg. 2018. Linux Performance. http://www.brendangregg. com/linuxperf.html.

[15] Prabhat K. Gupta. 2018. Xeon+FPGA Platform for the Data Center. https://www.ece.cmu.edu/ calcm/carl/lib/exe/fetch.php?media= carl15-gupta.pdf.

[16] Sangjin Han, Keon Jang, Aurojit Panda, Shoumik Palkar, Dongsu Han, and Sylvia Ratnasamy. 2015. SoftNIC: A Software NIC to Augment Hardware. In Technical Report No. UCB/EECS-2015-155. University of California at Berkeley.

[17] Sangjin Han, Keon Jang, KyoungSoo Park, and Sue Moon. 2010. PacketShader: A GPU-accelerated Software Router. In Special Interest Group on Data Communication (SIGCOMM). ACM.

[18] Intel. 2014. Xeon Phi Coprocessor System Software Developers Guide. https://software.intel.com/sites/default/files/managed/09/ 07/xeon-phi-coprocessor-system-software-developers-guide.pdf.

[19] Intel. 2018. $8259910 \mathrm{GbE}$ Controller Datasheet. https: //www.intel.com/content/dam/www/public/us/en/documents/ datasheets/82599-10-gbe-controller-datasheet.pdf.

[20] Intel. 2018. Data Direct I/O technology (Intel DDIO): a primer. http://www.intel.co.uk/content/dam/www/public/us/en/ documents/technology-briefs/data-direct-i-o-technology-brief.pdf.

[21] Intel. 2018. Intel VTune Amplifier 2017. https://software.intel.com/ en-us/intel-vtune-amplifier-xe.

[22] Intel. 2018. Virtualization technology for directed I/O. http://www.intel.co.uk/content/dam/www/public/us/en/documents/ product-specifications/vt-directed-io-spec.pdf.

[23] Norman P. Jouppi, Cliff Young, Nishant Patil, David Patterson, Gaurav Agrawal, Raminder Bajwa, Sarah Bates, Suresh Bhatia, Nan Boden, Al Borchers, Rick Boyle, Pierre-luc Cantin, Clifford Chao, Chris Clark, Jeremy Coriell, Mike Daley, Matt Dau, Jeffrey Dean, Ben Gelb, Tara Vazir Ghaemmaghami, Rajendra Gottipati, William Gulland, Robert Hagmann, C. Richard Ho, Doug Hogberg, John Hu, Robert Hundt, Dan Hurt, Julian Ibarz, Aaron Jaffey, Alek Jaworski, Alexander Kaplan, Harshit Khaitan, Daniel Killebrew, Andy Koch, Naveen Kumar, Steve Lacy, James Laudon, James Law, Diemthu Le, Chris Leary, Zhuyuan Liu, Kyle Lucke, Alan Lundin, Gordon MacKean, Adriana Maggiore, Maire Mahony, Kieran Miller, Rahul Nagarajan, Ravi Narayanaswami, Ray Ni, Kathy Nix, Thomas Norrie, Mark Omernick, Narayana Penukonda, Andy Phelps, Jonathan Ross, Matt Ross, Amir Salek, Emad Samadiani, Chris Severn, Gregory Sizikov, Matthew Snelham, Jed Souter, Dan Steinberg, Andy Swing, Mercedes Tan, Gregory Thorson, Bo Tian, Horia Toma, Erick Tuttle, Vijay Vasudevan, Richard Walter, Walter Wang, Eric Wilcox, and Doe Hyun Yoon. 2017. In-Datacenter Performance Analysis of a Tensor Processing Unit. In International Symposium on Computer Architecture (ISCA). ACM/IEEE.

[24] Anuj Kalia, Michael Kaminsky, and David G. Andersen. 2016. Design Guidelines for High Performance RDMA Systems. In Annual Technical Conference (ATC). USENIX.

[25] Thomas Karagiannis, Richard Mortier, and Antony Rowstron. 2008. Network Exception Handlers: Host-network Control in Enterprise Networks. In Special Interest Group on Data Communication (SIGCOMM). ACM.

[26] Antoine Kaufmann, SImon Peter, Naveen Kr. Sharma, Thomas Anderson, and Arvind Krishnamurthy. 2016. High Performance Packet Processing with FlexNIC. In International Conference on Architectural Support for Programming Languages and Operating Systems (ASPLOS). ACM.

[27] Matthew J. Koop, Wei Huang, Karthik Gopalakrishnan, and Dhabaleswar K. Panda. 2008. Performance analysis and evaluation of PCIe 2.0 and quad-data rate InfiniBand. In Symposium on High Performance Interconnects (HOTI). IEEE.

[28] Christoph Lameter. 2013. NUMA (Non-Uniform Memory Access): an overview. In acmqueue. ACM.

[29] Yanfang Le, Hyunseok Chang, Sarit Mukherjee, Limin Wang, Aditya Akella, Michael Swift, and T.V. Lakshman. 2017. UNO: Unifying Host and Smart NIC Offload for Flexible Packet Processing. In Symposium on Cloud Computing (SoCC). ACM.

[30] Ki Suh Lee, Han Wang, and Hakim Weatherspoon. 2013. SoNIC: Precise Realtime Software Access and Control of Wired Networks. In Networked Systems Design and Implementation (NSDI). USENIX Association.

[31] Bojie Li, Zhenyuan Ruan, Wencong Xiao, Yuanwei Lu, Yongqiang Xiong, Andrew Putnam, Enhong Chen, and Lintao Zhang. 2017. 
KV-Direct: High-Performance In-Memory Key-Value Store with Programmable NIC. In Symposium on Operating Systems Principles (SOSP). ACM.

[32] Sheng Li, Hyeontaek Lim, Victor W. Lee, Jung Ho Ahn, Anuj Kalia, Michael Kaminsky, David G. Andersen, O. Seongil, Sukhan Lee, and Pradeep Dubey. 2015. Architecting to Achieve a Billion Requests Per Second Throughput on a Single Key-value Store Server Platform. In International Symposium on Computer Architecture (ISCA). ACM.

[33] Tan Li, Yufei Ren, Dantong Yu, Shudong Jin, and Thomas Robertazzi. 2013. Characterization of Input/Output bandwidth performance models in NUMA architecture for data intensive applications. In International Conference on Parallel Processing (ICPP). IEEE.

[34] Hyeontaek Lim, Dongsu Han, David G. Andersen, and Michael Kaminsky. 2014. MICA: A Holistic Approach to Fast In-Memory Key-Value Storage. In Networked Systems Design and Implementation (NSDI). USENIX.

[35] Jiuxing Liu, Amith Mamidala, Abhinav Vishnn, and Dhabaleswar K. Panda. 2004. Performance evaluation of InfiniBand with PCI Express. In Symposium on High Performance Interconnects (HOTI). IEEE.

[36] K. Lostrie, P. De Meulenaere, M. Temmerman, N. Van Remortel, and W. Beaumont. 2013. Benchmarking of PCIe-performance in microTCAequipment. In International Conference on P2P, Parallel, Grid, Cloud and Internet Computing (3PGCIC). IEEE.

[37] Ilias Marinos, Robert Watson, and Mark Handley. 2014. Network Stack Specialization for Performance. In Special Interest Group on Data Communication (SIGCOMM). ACM.

[38] Ilias Marinos, Robert N.M. Watson, Mark Handley, and Randall R. Stewart. 2017. Disk|Crypt|Net: Rethinking the Stack for High-performance Video Streaming. In Special Interest Group on Data Communication (SIGCOMM). ACM.

[39] Larry McVoy and Carl Staelin. 1996. Lmbench: portable tools for performance analysis. In Annual Technical Conference (ATC). USENIX.

[40] Mellanox. 2018. Programmable ConnectX-3 Pro Adapter Card. https://www.mellanox.com/related-docs/prod_adapter_cards/PB_ Programmable_ConnectX-3_Pro_Card_VPI.pdf.

[41] David J. Miller, Philip M. Watts, and Andrew W. Moore. 2009. Motivating future interconnects: a differential measurement analysis of PCI latency. In Symposium on Architectures for Networking and Communications Systems (ANCS). ACM.

[42] L. Moll and M. Shand. 1997. Systems performance measurement on PCI pamette. In Symposium on FPGA-Based Custom Computing Machines (FCCM). IEEE.

[43] NetFPGA. 2018. Official website. https://www.netfpga.org.

[44] Netronome. 2018. NFP-4000 theory of operation. https: //www.netronome.com/static/app/img/products/silicon-solutions/ WP_NFP4000_TOO.pdf.

[45] Netronome. 2018. Product Brief: NFP-6000 intelligent Ethernet controller family. https://www.netronome.com/static/app/img/products/ silicon-solutions/PB_NFP6000.pdf.

[46] ntop. 2018. PF_RING repository. https://github.com/ntop/PF_RING. git.

[47] PCI-SIG. 2014. PCI Express Base Specification Revision 3.1.

[48] Omer Peleg, Adam Morrison, Benjamin Serebrin, and Dan Tsafrir. 2015. Utilizing the IOMMU Scalably. In Annual Technical Conference (ATC). USENIX.

[49] Ben Pfaff, Justin Pettit, Teemu Koponen, Keith Amidon, Martin Casado, and Scott Shenker. 2009. Extending Networking into the Virtualization Layer. In Hot Topics in Networks (HotNets). ACM.

[50] Ian Pratt and Keir Fraser. 2001. Arsenic: A user-accessible gigabit ethernet interface. In International Conference on Computer Communications (INFOCOM). IEEE.
[51] Andrew Putnam, Adrian M Caulfield, Eric S Chung, Derek Chiou, Kypros Constantinides, John Demme, Hadi Esmaeilzadeh, Jeremy Fowers, Gopi Prashanth Gopal, Jan Gray, et al. 2014. A reconfigurable fabric for accelerating large-scale datacenter services. In International Symposium on Computer Architecture (ISCA). ACM/IEEE.

[52] Sivasankar Radhakrishnan, Yilong Geng, Vimalkumar Jeyakumar, Abdul Kabbani, George Porter, and Amin Vahdat. 2014. SENIC: Scalable NIC for End-host Rate Limiting. In Conference on Networked Systems Design and Implementation (NSDI). USENIX.

[53] Kaushik Kumar Ram, Jayaram Mudigonda, Alan L. Cox, Scott Rixner, Parthasarathy Ranganathan, and Jose Renato Santos. 2010. sNICh: Efficient Last Hop Networking in the Data Center. In Architectures for Networking and Communications Systems (ANCS). ACM.

[54] Luigi Rizzo. 2012. netmap: a novel framework for fast packet I/O. In Annual Technical Conference (ATC). USENIX.

[55] Arjun Roy, Hongyi Zeng, Jasmeet Bagga, George Porter, and Alex C. Snoeren. 2015. Inside the Social Network's (Datacenter) Network. In Special Interest Group on Data Communication (SIGCOMM). ACM.

[56] Alan Shieh, Srikanth Kandula, and Emin Gun Sirer. 2010. SideCar: Building Programmable Datacenter Networks Without Programmable Switches. In Hot Topics in Networks (HotNets). ACM.

[57] Edward Solari and Brad Congdon. 2003. The Complete PCI Express Reference.

[58] Robert Soulé, Shrutarshi Basu, Robert Kleinberg, Emin Gün Sirer, and Nate Foster. 2013. Managing the Network with Merlin. In Hot Topics in Networks (HotNets). ACM.

[59] Gavin Stark and Sakir Sezer. 2013. NFP-6xxx - A 22nm highperformance network flow processor for $200 \mathrm{~Gb} / \mathrm{s}$ Software Defined Networking. In Symposium on High Performance Chips (Hot Chips). IEEE/ACM.

[60] T. von Eicken, A. Basu, V. Buch, and W. Vogels. 1995. U-Net: A Userlevel Network Interface for Parallel and Distributed Computing. In Symposium on Operating Systems Principles (SOSP). ACM.

[61] Jose Fernando Zazo, Sergio Lopez-Buedo, Yury Audzevich, and Andrew W. Moore. 2015. A PCIe DMA engine to support the virtualization of $40 \mathrm{Gbps}$ FPGA-accelerated network appliances. In International Conference on Reconfigurable Computing and FPGAs (RECONFIG). IEEE.

[62] Noa Zilberman, Yury Audzevich, Adam G. Covington, and Andrew W. Moore. 2014. NetFPGA SUME: toward 100 Gbps as research commodity. In Micro. IEEE.

[63] Noa Zilberman, Matthew Grosvenor, Diana-Andreea Popescu, Neelakandan Manihatty-Bojan, Gianni Antichi, Marcin Wojcik, and Andrew W. Moore. 2017. Where Has My Time Gone?. In Passive and Active Measurement (PAM). Springer. 